\title{
GeRminaÇÃo DE SEMENTES DE Melaleuca quinquenervia EM Condições de EsTResse HídRICo E SAlino ${ }^{1}$
}

\author{
Germination of Melaleuca quinquenervia Seeds under Water and Salt Stress Conditions
}

\author{
MARTINS, C.C. ${ }^{2}$, PEREIRA, M.R.R. ${ }^{2}$ e MARCHI, S.R. ${ }^{3}$
}

\begin{abstract}
RESUMO - O comportamento invasivo de Melaleuca quinquenervia em áreas úmidas deve-se à sua estratégia de regeneração agressiva, que está alicerçada na produção de sementes em massa. O conhecimento da fisiologia da germinação de sementes de plantas daninhas pode contribuir significativamente para o desenvolvimento de estratégias de manejo. O objetivo deste trabalho foi avaliar os possiveis efeitos dos estresses hídrico e salino na germinação de sementes de $M$. quinquenervia. A semeadura foi realizada com quatro repetições de $0,05 \mathrm{~g}$ de sementes em papel umedecido com soluções nos potenciais osmóticos de 0,$0 ;-0,2 ;-0,4$; e -0,8 MPa, induzidos com polietilenoglicol (PEG 6000) e NaCl. O teste de germinação foi conduzido a $25^{\circ} \mathrm{C}$ na presença de luz. Avaliou-se a primeira contagem do teste aos sete dias após a semeadura e, semanalmente, a germinação (plântulas normais) até os 28 dias. Foi calculado o indice de velocidade de germinação. A análise dos resultados permitiu a conclusão de que o estresse hídrico acarreta maior redução na velocidade de germinação e na germinação acumulada de sementes de $M$. quinquenervia do que o estresse salino e, independentemente da substância utilizada para indução do estresse, o limite para germinação está entre -0,4 e $-0,8 \mathrm{MPa}$.
\end{abstract}

Palavras-chave: estresse osmótico, planta daninha, potencial osmótico, salinidade, vigor.

\begin{abstract}
The invasive behavior of Melaleuca quinquenervia in wetlands seems attributable to its inherently aggressive regeneration strategies associated to massive seed production. The knowledge of the germination physiology of weed seeds can contribute significantly to the development of strategies for their management. This work aimed to evaluate the possible effects of water and salt stress on the germination of $\boldsymbol{M}$. quinquenervia seeds. Four replications with $0.05 \mathrm{~g}$ of seeds were sown in paper moistened with solutions with the potential of $0.0 ;-0.2 ;-0.4$ and $-0.8 \mathrm{MPa}$, induced with polyethylene glycol (PEG 6000) and NaCl. Germination test was carried out at $25{ }^{\circ} \mathrm{C}$ in the presence of light. The percentage of normal seedlings was obtained seven days after sowing (first count) and, weekly, up to 28 days. The speed of germination index was calculated. The results allowed to conclude that water stress causes a greater reduction in germination speed and accumulated germination of $\boldsymbol{M}$. quinquenervia seeds than saline stress, and that, regardless of the substance used for stress induction, germination limit is between -0.4 and $-0.8 \mathrm{MPa}$.
\end{abstract}

Keywords: osmotic stress, weed, osmotic potential, salinity, vigor.

\section{INTRODUÇÃO}

A Melaleuca quinquenervia é uma árvore exótica invasora originária da Austrália e pertencente à família Myrtaceae, adaptada tanto a áreas secas como alagadas. A espécie foi introduzida nos Estados Unidos no início de 1886 como planta ornamental e também para ajudar a secar áreas pantanosas (Dray Jr. et al., 2004). Devido à agressividade, adaptação a ambientes úmidos e difícil controle, tornou-se uma planta invasora dominante em

1 Recebido para publicação em 14.1.2011 e na forma revisada em 18.2.2011.

2 Profa. Dra., FATEC - Silvicultura, Rua Amantino de Oliveira Ramos no 60, 18300-000 Capão Bonito-SP, Brasil, <cibele@fca.unesp.br>; <mariarenata10@hotmail.com>; ${ }^{3}$ Professor Adjunto, ICET/UFMT, Barra do Garça-MT, $<$ sidneimarchi.ufmt@gmail.com>. 
pântanos da Flórida do sul, predominando no ecossistema dos Everglades. Nessas regiões, plantas de melaleuca ocupam grandes áreas e têm representado uma séria ameaça para as comunidades naturais (Serbesoff-King, 2003; Dray Jr. et al., 2004).

As árvores proliferam-se rapidamente, por produzirem e armazenarem grande quantidade de sementes; uma única planta é capaz de produzir até um milhão de mudas por ano. As árvores são altamente inflamáveis e, após estresse causado por fogo ou geada, a produção de sementes é intensificada (Doran \& Turnbull, 1997; Serbesoff-King, 2003).

As sementes são dispersas pelo vento e água e, rapidamente, capazes de estabelecer novos estandes a longas distâncias (Bodle et al., 1994). Os frutos de melaleuca são cápsulas lenhosas, cilindricas ou quadradas, e cada cápsula contém de 200 a 300 sementes. As sementes de melaleuca apresentam tamanho e peso reduzido: em um grama de sementes podem-se contabilizar 30 mil sementes; destas, mais de 2.650 são viáveis, com dimensões de $1 \mathrm{~mm}$ de comprimento e $0,1 \mathrm{~mm}$ de largura (Woodall, 1983; Bodle et al., 1994; Van et al., 2005). As pequenas dimensões dificultam a individualização das sementes para estudos de germinação, de modo semelhante ao verificado para sementes de eucalipto (Brasil, 2009).

As sementes são viáveis e capazes de germinar após cinco dias da semeadura em substrato umedecido e mantido a aproximadamente $30^{\circ} \mathrm{C}$; as sementes podem apresentar dormência, porém em níveis que não afetam significativamente a germinação (Rayachhetry et al., 1998; Serbesoff-King, 2003; Van et al., 2005). Durante os meses frescos e secos de inverno, típicos do sul da Flórida, as sementes podem não germinar imediatamente (Van et al., 2005). A sobrevivência e capacidade de germinação de sementes em condições adversas são fontes importantes para a regeneração de plantas e competição (Murdoch \& Ellis, 1992).

As plantas de melaleuca são adaptadas a solos encharcados, ácidos e moderadamente salinos (Woodall, 1983; Doran \& Turnbull, 1997; Kaufman \& Smouse, 2001); contudo, não foram encontrados dados sobre a germinação de sementes dessa espécie em condições de estresse hídrico e salino.

Estudos sobre relações hídricas são importantes para o conhecimento da biologia das sementes de plantas daninhas (Yamashita et al., 2009; Ghaderi-Far et al., 2010). Alguns trabalhos têm sido conduzidos utilizando soluções com diferentes potenciais osmóticos para umedecer substratos, normalmente papel, no qual as sementes são colocadas para germinar, procurando simular condições de baixa disponibilidade de água no solo (Yamashita et al., 2009; Ghaderi-Far et al., 2010). Esses trabalhos visam simplificar as condições complexas observadas nas avaliações em campo ou em casa de vegetação (Santos et al., 1992; Ghaderi-Far et al., 2010).

Entre as diversas soluções usadas para simular condições de estresse hídrico, a solução de polietilenoglicol (PEG) tem sido comumente utilizada, pois esse agente osmótico é quimicamente inerte, não tóxico e não eletrolítico (Marcos Filho, 2005). O polietilenoglicol não penetra nas células, devido ao seu elevado peso molecular (Hasegawa et al., 1984).

Na determinação da tolerância das plantas ao estresse salino, um dos métodos mais difundidos é a observação da capacidade germinativa das sementes nessas condições (Larcher, 2000), pois a salinidade afeta negativamente o crescimento e o desenvolvimento das plantas, e seus efeitos dependem da espécie vegetal. $O$ excesso de sais solúveis provoca redução do potencial hídrico do solo, induzindo uma menor capacidade de absorção de água pelas plantas. A redução do potencial hídrico, associada ao efeito tóxico dos sais, interfere inicialmente no processo de absorção de água pelas sementes, o que pode influenciar na germinação, no vigor das plântulas e, consequentemente, no desenvolvimento normal das plantas (Marcos Filho, 2005; Yamashita et al., 2009; GhaderiFar et al., 2010).

Considerando-se a introdução da melaleuca em projetos paisagísticos no Brasil, a agressividade da espécie e a existência de regiões ecologicamente favoráveis ao seu desenvolvimento em nosso país, com grandes extensões alagadas de água doce e salobra, este trabalho foi realizado com o objetivo de 
avaliar o efeito dos estresses hídrico e salino na germinação de sementes dessa espécie.

\section{MATERIAL E MÉTODOS}

O experimento foi conduzido no Laboratório de Análise de Sementes da Faculdade de Tecnologia em Silvicultura de Capão Bonito (FATEC), do Estado de São Paulo. Os frutos foram colhidos em 15 árvores utilizadas na arborização de ruas em condomínio localizado na cidade de Barueri-SP, a partir da escalada das árvores matrizes e do corte de ramos com cápsulas maduras: com coloração amarronzada e fendas axiais demarcadas. Após a colheita, os ramos foram levados para barracão coberto, onde permaneceram até a secagem e abertura das cápsulas. Os ramos foram agitados sobre bandeja, para liberação e recolhimento das sementes.

No teste de germinação, a semeadura seguiu a metodologia recomendada para Eucalyptus spp., utilizando-se o peso de sementes (Brasil, 2009). Assim, o teste de germinação foi conduzido com quatro subamostras de $0,05 \mathrm{~g}$ de sementes, distribuídas uniformemente sobre duas folhas de papel mata-borrão umedecidas com soluções de polietilenoglicol (PEG) 6000 e cloreto de sódio $(\mathrm{NaCl})$ nos potenciais osmóticos de $0 ;-0,2 ;-0,4 ;$ e - - , $8 \mathrm{MPa}$, na quantidade de 2,5 vezes a massa do papel (Brasil, 2009). No nivel zero foi utilizada apenas água destilada, para umedecer o substrato. A quantidade de soluto utilizada na preparação das soluções de diferentes potenciais osmóticos está apresentada na Tabela 1.

Cada subamostra do teste de germinação foi acondicionada em caixas plásticas transparentes $(11,0 \times 11,0 \times 3,5 \mathrm{~cm})$, colocadas em sacos plásticos de $0,05 \mathrm{~mm}$ de espessura, para conservação da umidade do substrato, e mantidas a $25^{\circ} \mathrm{C}$ e $8 \mathrm{~h}$ de luz. Foram consideradas plântulas normais aquelas com comprimento minimo de $0,3 \mathrm{~mm}$, sendo as contagens realizadas diariamente de 7 até 28 dias após a semeadura, e os resultados foram expressos em número de plântulas por 0,05 de sementes (Brasil, 2009). A primeira contagem de germinação e o índice de velocidade de germinação foram realizados em conjunto com o teste de germinação: no teste da primeira contagem foram contabilizadas as plântulas normais presentes no sétimo dia após a semeadura, e na determinação do índice de velocidade de germinação (IVG) foi utilizada a metodologia estabelecida por Maguire (1962).

O experimento foi conduzido no delineamento inteiramente casualizado, com os tratamentos em arranjo fatorial $2 \times 4$ (agente osmótico x potencial osmótico), e quatro repetições; a comparação entre as médias foi realizada por meio do teste de Tukey a $5 \%$ de probabilidade. As médias apresentadas nas tabelas são de dados não transformados, os quais também foram submetidos à análise de regressão polinomial; foi selecionado o modelo significativo de maior ordem $\left(R^{2}\right)$, empregando-se a equação que melhor se ajustou aos dados.

\section{RESULTADOS E DISCUSSÃO}

Para germinação, primeira contagem e índice de velocidade de germinação, a comparação dos valores obtidos dentro de cada nivel de potencial osmótico avaliado permitiu constatar que não houve influência entre os agentes osmóticos PEG e $\mathrm{NaCl}$, nem interação entre os fatores agentes osmóticos e potencial osmótico (Tabela 2).

De modo geral, com o aumento dos potenciais osmóticos avaliados, verificou-se efeito mais intenso e prejudicial ao desempenho das sementes submetidas ao estresse hídrico (PEG) do que ao estresse salino $(\mathrm{NaCl})$, mais evidente nos níveis intermediários de -0,2 e $-0,4 \mathrm{MPa}$ (Tabela 2 e Figuras 1 e 2). Em condições de estresse hídrico, a velocidade de germinação foi significativa e progressivamente reduzida a partir de potenciais de $-0,2 \mathrm{MPa}$, enquanto em condições de estresse hídrico isso só foi observado a partir de -0,4 MPa.

Tabela 1 - Quantidade de soluto utilizado na preparação das soluções de diferentes potenciais osmóticos com PEG 6000 e $\mathrm{NaCl}$ a $25^{\circ} \mathrm{C}$

\begin{tabular}{|c|c|c|}
\hline $\begin{array}{c}\text { Potencial osmótico } \\
\text { estimado (MPa) }\end{array}$ & $\begin{array}{c}\text { Concentração } \\
\left(\mathrm{g} \text { PEG } 6000 \mathrm{~L}^{-1} \mathrm{H}_{2} \mathrm{O}\right)\end{array}$ & $\begin{array}{c}\text { Concentração } \\
\left(\mathrm{g} \mathrm{NaCl} \mathrm{L}^{-1} \mathrm{H}_{2} \mathrm{O}\right)\end{array}$ \\
\hline 0,0 & 0,00 & 0,00 \\
\hline$-0,2$ & $119,571(11 \%)$ & $4,730(0,4 \%)$ \\
\hline$-0,4$ & $178,343(17 \%)$ & $9,460(0,9 \%)$ \\
\hline$-0,8$ & $261,948(26 \%)$ & $18,920(1,9 \%)$ \\
\hline
\end{tabular}


Tabela 2 - Germinação, teste da primeira contagem e índice de velocidade de germinação (IVG) de sementes deMelaleuca quinquenervia submetidas a diferentes potenciais osmóticos induzidos por PEG 6000 e $\mathrm{NaCl}$

\begin{tabular}{|c|c|c|c|c|c|c|}
\hline \multirow{2}{*}{$\begin{array}{c}\text { Potencial osmótico } \\
(\mathrm{MPa})\end{array}$} & \multicolumn{2}{|c|}{ Germinação } & \multicolumn{2}{|c|}{ Primeira contagem } & \multicolumn{2}{|c|}{ IVG } \\
\hline & PEG & $\mathrm{NaCl}$ & PEG & $\mathrm{NaCl}$ & PEG & $\mathrm{NaCl}$ \\
\hline 0,0 & $72,5 \mathrm{a}^{\mathrm{1}^{/ /}}$ & $72,5 \mathrm{a}$ & $59,0 \mathrm{a}$ & $59,0 \mathrm{a}$ & $9,369 \mathrm{a}$ & $9,369 \mathrm{a}$ \\
\hline$-0,2$ & $67,5 \mathrm{a}$ & $69,5 \mathrm{a}$ & $43,8 \mathrm{~b}$ & $46,0 \mathrm{~b}$ & $7,857 \mathrm{~b}$ & $8,214 \mathrm{a}$ \\
\hline$-0,4$ & $45,0 \mathrm{~b}$ & $58,8 \mathrm{a}$ & $0,0 \mathrm{c}$ & $10,5 \mathrm{c}$ & $3,089 \mathrm{c}$ & $4,798 \mathrm{~b}$ \\
\hline$-0,8$ & $0,0 \mathrm{c}$ & $0,0 \mathrm{~b}$ & $0,0 \mathrm{c}$ & $0,0 \mathrm{~d}$ & $0,000 \mathrm{~d}$ & $0,000 \mathrm{c}$ \\
\hline F Potencial Osmótico (P) & \multicolumn{2}{|c|}{68,703} & \multicolumn{2}{|c|}{215,543} & \multicolumn{2}{|c|}{148,763} \\
\hline $\mathrm{F}$ Agentes Osmóticos(A) & \multicolumn{2}{|c|}{$0,957^{\mathrm{ns}}$} & \multicolumn{2}{|c|}{$2,581^{\mathrm{ns}}$} & \multicolumn{2}{|c|}{$2,613^{\mathrm{ns}}$} \\
\hline$F_{P x A}$ & \multicolumn{2}{|c|}{$0,674^{\mathrm{ns}}$} & \multicolumn{2}{|c|}{$1,581^{\mathrm{ns}}$} & \multicolumn{2}{|c|}{$1,065^{\mathrm{ns}}$} \\
\hline CV $(\%)$ & \multicolumn{2}{|c|}{23,6} & \multicolumn{2}{|c|}{20,6} & \multicolumn{2}{|c|}{18,4} \\
\hline
\end{tabular}

1/ Médias seguidas pela mesma letra na coluna não diferem estatisticamente pelo teste de Tukey a $5 \%$ de probabilidade; significativo a $1 \%$ de probabilidade; ${ }^{\text {ns }}$ não significativo.

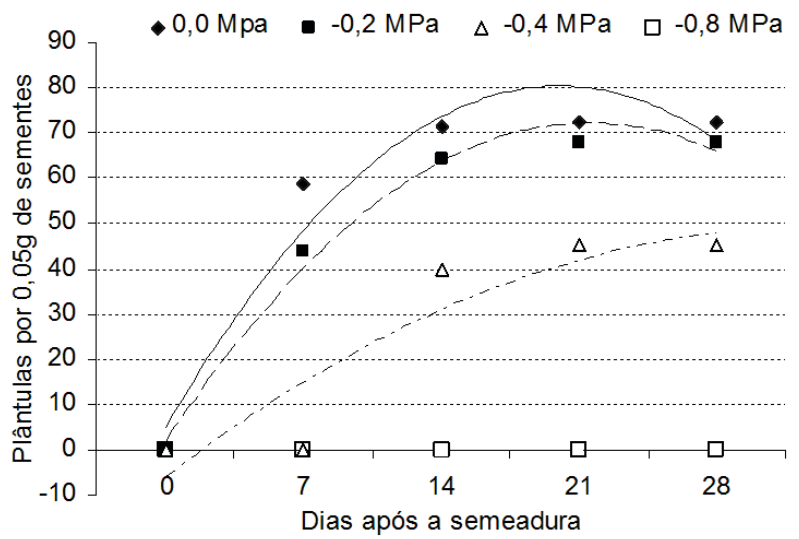

Figura 1 - Germinação acumulada ao longo do tempo de sementes de Melaleuca quinquenervia submetidas a estresse hídrico induzido por PEG 6000 nos potenciais osmóticos de $0,0 \mathrm{MPa}\left(\mathrm{y}=-57,2+71,35 \mathrm{x}-9,25 \mathrm{x}^{2} ; \mathrm{R}^{2}=0,945\right) ;-0,02 \mathrm{MPa}$ $\left(\mathrm{y}=-51,2+60,57 \mathrm{x}-7,43 \mathrm{x}^{2} ; \mathrm{R}^{2}=0,988\right) ; \mathrm{y}-0,04 \mathrm{MPa}$ $\left(y=-32+28,5 x-2,5 x^{2} ; R^{2}=0,841\right) ;$ e $-0,08 \mathrm{MPa}$.

Os valores do teste da primeira contagem de germinação (Tabela 2) permitem verificar que o estresse promovido por ambos os agentes osmóticos prejudicou o vigor de forma significativa e progressiva a partir do potencial osmótico de - 0,2 MPa, embora em condições de estresse hídrico o efeito tenha sido mais drástico que sob estresse salino, pois a partir de -0,4 MPa nenhuma plântula de melaleuca foi contabilizada no substrato umedecido com solução de PEG, enquanto aproximadamente 10 sementes germinaram com solução salina.

A germinação acumulada das sementes devido ao estresse hídrico promovido pelo PEG foi significativa e progressivamente reduzida

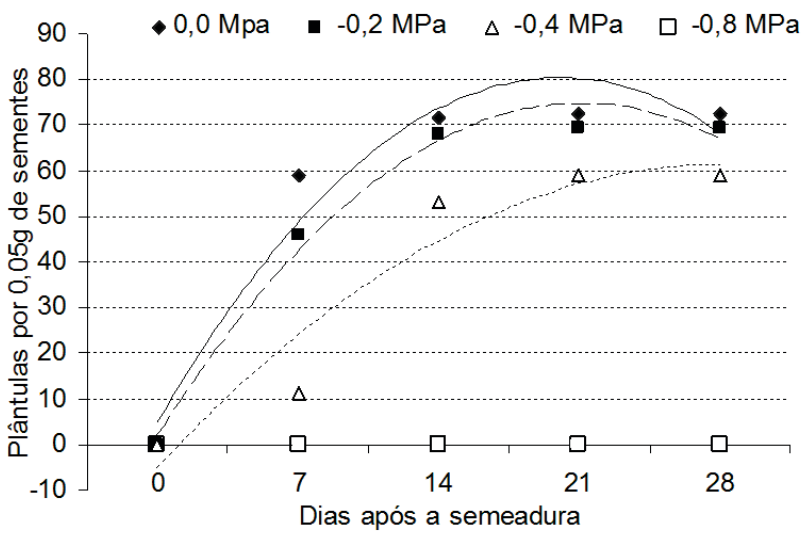

Figura 2 - Germinação acumulada ao longo do tempo de sementes de Melaleuca quinquenervia submetidas a estresse hídrico induzido por $\mathrm{NaCl}$ nos potenciais osmóticos de $0,0 \mathrm{MPa}$ $\left(\mathrm{y}=-57,2+71,35 \mathrm{x}-9,25 \mathrm{x}^{2} ; \mathrm{R}^{2}=0,945\right) ;-0,02 \mathrm{MPa}$ $\left(\mathrm{y}=-54,4+64,464 \mathrm{x}-8,0357 \mathrm{x}^{2} ; \mathrm{R}^{2}=0,985\right) ;-0,04 \mathrm{MPa}$ $\left(y=-42,4+41,457 x-4,1429 x^{2} ; R^{2}=0,917\right) ;$ e $-0,08 \mathrm{MPa}$.

a partir do potencial osmótico de $-0,4 \mathrm{MPa}$, ao passo que em condições de estresse salino somente foi constatada redução significativa da germinação a -0,8M Pa. Nesse último potencial osmótico obtido com ambos os agentes osmóticos (PEG e $\mathrm{Na} \mathrm{C1}$ ), verificou-se que não houve mais germinação (Tabela 2 e Figuras 1 e 2). Assim, constatou-se que, de modo independente da substância utilizada para indução do estresse, o potencial osmótico limite para a germinação estaria situado entre $-0,4$ e $-0,8 \mathrm{MPa}$.

Os resultados indicaram que a velocidade de germinação e o vigor das sementes avaliado pelo teste da primeira contagem de 
M. quinquenervia foram mais afetados pelos diferentes niveis de potenciais osmóticos impostos que a porcentagem de germinação. O potencial hídrico altera o alongamento celular e a sintese de parede durante o processo de germinação (Marcos Filho, 2005). Esse fato vem corroborar relatos de que o aumento do estresse ambiental, em geral, leva inicialmente a um decréscimo na velocidade de germinação e só posteriormente vem afetar a germinabilidade das sementes (Marcos Filho, 2005).

Para cada espécie invasora existe um valor de potencial hídrico crítico, abaixo do qual a germinação não ocorre. Conyza canadensis (Nandula et al., 2006) apresenta redução na germinação sob potencial osmótico inferior a -0,8 MPa, enquanto para Leucaena leucocephala e Senna occidentalis esse valor seria de -0,4 MPa (Borges et al., 1997; Norsworthy \& Oliveira, 2005); para Campsis radicans e Solanum sarrachoides, potenciais osmóticos menores que $-0,2 \mathrm{MPa}$ afetam a germinação (Chachalis \& Reddy, 2000; Zhou et al., 2005). Entre essas espécies invasoras, as sementes de Emilia sonchifolia e Melilotus officinalis foram as mais sensiveis ao estresse hídrico, apresentando reduções de germinação em potenciais osmóticos inferiores a $-0,1 \mathrm{MPa}$ (Ghaderi-Far et al., 2010; Yamashita et al., 2009).

A análise dos resultados permitiu a conclusão de que o estresse hídrico acarreta maior redução na velocidade de germinação e na germinação acumulada de sementes de $M$. quinquenervia do que o estresse salino; independentemente da substância utilizada para indução do estresse, o limite para germinação está entre -0,4 e -0,8 MPa.

\section{LITERATURA CITADA}

BODLE, M. J.; FERRITER, A. P.; THAYER, D. D. The biology, distribution, and ecological consequences of Melaleuca quinquenervia in the Everglades. In: DAVIS, S. M.; OGDEN, J.C. (Eds.). Everglades: the ecosystem and its restoration. St. Lucie: Press, Delray Beach, 1994. p. 341-355.

BORGES, E. E. L.; BORGES, R. C. G; PAULA, N. F. Efeito da temperatura e do estresse hídrico na germinação de sementes de fedegoso (Senna macranthera (Collad.) Irwin et Barn.) e de Leucaena leucocephala (Lam.) de Wit. R. Bras. Sementes, v. 19, n. 2, p. 155-158, 1997.
BRASIL. Ministério da Agricultura, Pecuária e Abastecimento. Secretaria de Defesa Agropecuária. Regras para análise de sementes. Brasília: 2009. 399 p.

CHACHALIS, D.; REDDY, K. N. Factors affecting Campsis radicans seed germination and seedling emergence. Weed Sci., v. 48, n. 2 , p. $212-216,2000$

DORAN, J. C.; TURNBULL, J. W. Australian trees and shrubs: species for land rehabilitation and farm planting in the tropics. Canberra: Australian Centre for International Agricultural Research, 1997. 384 p. Disponível em <HTTP:// www.aciar.gov.au/publication/MN024> . Acesso em 04 fev. de 2010 .

DRAY Jr., F.A. et al. Genetic variation in melaleuca quinquenervia affects the biocontrol agent oxyops vitiosa. Weed Technol., v. 18, n. 1, p. 1400-1402, 2004.

GHADERI-FAR, F.; GHEREKHLOO, J.; ALIMAGHAM, $M$. Influence of environmental factors on seed germination and seedling emergence of yellow sweet clover (Melilotus officinalis). Planta Daninha, v. 28, n. 3, p. 436-469, 2010.

HASEGAWA, P. M. et al. Cellular mechanisms of tolerance to water stress. HortScience, v. 19, n. 3, p. 371-377, 1984.

KAUFMAN, S. R.; SMOUSE, P. E. Comparing indigenous and introduced populations of Melaleuca quinquenervia (Cav.) Blake: response of seedlings to water and $\mathrm{pH}$ levels Oecologia, v. 127, n. 3, p. 487-494, 2001.

LARCHER, W. Ecofisiologia vegetal. São Carlos: Rima, 2000. 531 p.

MAGUIRE, J. D. Speed of germination and in selection and evaluation for seedling emergence and vigor. Crop Sci., v. 2, n. 1, p. 176-177, 1962.

MARCOS FILHO, J. Fisiologia de sementes de plantas cultivadas. Piracicaba: FEALQ, 2005. 495 p.

MURDOCH, A. J.; ELLIS, R. H. Longevity, viability, and dormancy. In: FENNER, M. (Ed.). Seeds: the ecology of regeneration in plant communities. Wallingford: $\mathrm{CAB}$ International, 1992. p. 193-229.

NANDULA, V. K. et al. Factors affecting germination of horseweed (Conyza canadensis). Weed Sci., v. 54, n. 5, p. 898-902, 2006.

NORSWORTHY, J. K.; OLIVEIRA, M. J. Coffee senna (Cassia occidentalis) germination and emergence is affected by environmental factors and seedling depth. Weed Sci., v. 53, n. 5 , p. $657-662,2005$

RAYACHHETRY, M. B.; VAN, T.K.; CENTER, T. D. Regeneration potential of the canopy-held seeds of Melaleuca quinquenervia in south Florida. Int. J. Plant Sci., v. 159, n. 3, p. 648-654, 1998. 
SANTOS, V. L. M. et al. Efeito do estresse hídrico e salino na germinação e vigor de sementes de soja. R. Bras. Sementes, v. 14, n. 2, p. 189-194, 1992

SERBESOFF-KING, K. Melaleuca in Florida: a literature review on the taxonomy, distribution, biology, ecology, economic importance and control measures. J. Aquat. Plant Manag., v. 41, n. 2, p. 98-112, 2003.

VAN, T. K.; RAYAMAJHI, M. B.; CENTER, T. D. Seed longevity of Melaleuca quinquenervia: a burial experiment in south Florida. J. Aquat. Plant Manag., v. 43, n. 1, p. 39-42, 2005.
WOODALL, S. L. Establishmentof Melaleuca quinquenervia seedlings in the pine-cypress ecotone of southwest Florida. Fla Sci., v. 46, n. 2, p. 65-72, 1983

YAMASHITA, O. M. et al. Fatores ambientais sobre a germinação de Emilia sonchifolia. Planta Daninha, v. 27, n. 4, p. 673-681, 2009.

ZHOU, J.; DECKARD, E. L.; AHRENS, W. H. Factors affecting germination of hairy nightshade (Solanum sarrachoides) seeds. Weed Sci., v. 53, n. 1, p. 41-45, 2005. 